\title{
ANÁLISE DOS MODELOS DE AVALIAÇÃO RESIDUAL INCOME VALUATION, ABNORMAL EARNINGS GROWTH E FLUXO DE CAIXA DESCONTADO APLICADOS ÀS OFERTAS PÚBLICAS DE AQUISIÇÃO DE AÇÕES NO BRASIL
}

\section{ANALYSIS OF RESIDUAL INCOME MODEL ASSESSMENT VALUATION, ABNORMAL EARNINGS GROWTH AND DISCOUNTED CASH FLOW APPLIED PUBLIC TENDER OFFER TO ACQUIRE SHARE IN BRAZIL}

\author{
José Elias Feres De Almeida ${ }^{a}$; Giovani Antonio Silva Brito ${ }^{b}$; \\ Flávio Donizete Batistella ${ }^{c}$; Eliseu Martins ${ }^{d}$ \\ a Doutor em Ciências Contábeis FEA/USP \\ Professor Adjunto do Departamento de Ciennicas Contábeis da UFES \\ ${ }^{b}$ Doutor em Ciências Contábeis FEA/USP \\ Professor da Fipecafi - Fundação Instituto de Pesquisas Contábeis, Atuariais e Financeiras \\ c Doutor em Ciências Contábeis FEA/USP \\ Professor da Faculdade de Economia, Administração e Contabilidade de Ribeirão Preto da Universidade de São Paulo \\ ${ }^{d}$ Livre-Docente e Doutor em Ciências Contábeis pela FEA-USP \\ Professor Emérito da Faculdade de Economia, Administração e Contabilidade da Universidade de São Paulo
}

\section{Resumo}

Uma companhia aberta, quando realiza uma Oferta Pública de Aquisição de Ações (OPA), contrata um agente especializado para elaborar o laudo de avaliação em conformidade com as disposições normativas. Este artigo examina 97 laudos de avaliação de OPAs divulgados no Brasil no período de 2000 a 2007. O modelo de avaliação mais utilizado e disponibilizado nos laudos é o Fluxo de Caixa Descontado. O objetivo desta pesquisa é examinar se os valores das empresas estimados por meio de modelos com maior embasamento na contabilidade, o Residual Income Valuation (RIV) e o Abnormal Earnings Growth (AEG), se aproximam dos valores estimados pelo modelo de Fluxo de Caixa Descontado, conforme constam dos laudos de avaliação. Os modelos RIV e AEG foram aplicados em amostras com 21 e 39 firmas, respectivamente. Os resultados dos testes realizados na amostra indicam que não há diferenças estatisticamente significantes entre os valores estimados pelos modelos RIV e AEG em relação aos valores estimados pelo modelo de Fluxo de Caixa Descontado.

Palavras-chave: Avaliação de Empresas; Laudos de Ofertas Públicas de Ações; Residual Income Valuation (RIV); Abnormal Earnings Growth (AEG); Fluxo de Caixa Descontado (FCD).

\begin{abstract}
A listed company, when it carries out a public tender offer to acquire shares (PTO), retains a specialized firm to conduct an appraisal of the target company, in conformity with applicable regulations. This article examines 97 appraisal reports of PTOs announced in Brazil over the period from 2000 to 2007. The valuation model most often used in these reports was discounted cash flow. Our aim is to examine whether the valuations estimated by models more closely based on accounting numbers, namely residual income valuation (RIV) and abnormal earnings growth (AEG), produce valuations near those estimated by the discounted cash flow method, according to the appraisal reports. We applied the RIV and AEG models to samples of 21 and 39 firms, respectively. The results of the tests performed indicate there are no statistically significant differences between the values estimated by either the RIV or AEG method in relation to those estimated by the discounted cash flow method.
\end{abstract}

Keywords: Valuation; Appraisal Reports of Public Tender Offer to Acquire Shares; Residual Income Valuation (RIV); Abnormal Earnings Growth (AEG); Discounted Cash Flow (FCD). 


\section{INTRODUÇÃ̃O}

Avaliação de empresas é um tema bastante pesquisado internacionalmente, no entanto, o Brasil ainda carece de estudos que se dediquem a testar as novas metodologias, os novos modelos e as novas abordagens que foram desenvolvidas na última década (OHLSON e LOPES, 2007).

O Fluxo de Caixa Descontado é um dos modelos mais utilizados e debatidos no âmbito acadêmico e no mercado financeiro, todavia, a literatura apresenta diversos outros modelos de avaliação de empresas que são alternativos ao Fluxo de Caixa Descontado. Os modelos com maior embasamento em dados contábeis, principalmente lucro e patrimônio líquido, ainda são pouco explorados em pesquisas acadêmicas e, menos ainda, utilizados pelo mercado no Brasil (OHLSON e LOPES, 2007; LOPES, 2001 e MARTINS, 2001).

Os modelos com maior embasamento na contabilidade são datados da década de 60 (EDWARDS e BELLS, 1961), no entanto, Ohlson (1995) desenvolveu toda a modelagem analítica para a sua validade, fato que disseminou a avaliação de empresas com base na contabilidade nos mercados desenvolvidos, principalmente nos Estados Unidos e Inglaterra.

Os modelos estruturados por Ohlson (1995), denominado Residual Income Valuation - RIV - (Avaliação pelos Lucros Residuais), e por Ohlson (2005) e Ohlson e Juettner-Nauroth (2005), denominado Abnormal Earnings Growth - AEG - (Avaliação pelo Crescimento dos Lucros Anormais), tiveram repercussão na pesquisa sobre avaliação de empresas. Penman (2005) apresentou a derivação e a convergência entre os modelos pelo que ele define como engenharia reversa, segundo a qual é possível avaliar as empresas utilizando dados contábeis, principalmente o lucro líquido e o patrimônio líquido, chegando aos mesmos valores pelos dois modelos.

Diversos estudos internacionais mostraram a superioridade dos modelos baseados em dados contábeis em relação aos demais (OHLSON e LOPES, 2007). No Brasil, ainda há poucas evidências a respeito da superioridade de um modelo em relação ao outro, podendo-se destacar os estudos de Lopes (2001), Famá e Leite (2003), Galdi e Lopes (2006) e Ferreira (2008).

Outro ponto debatido por pesquisadores é se os valores estimados pelos modelos com maior embasamento na contabilidade divergem dos valores estimados pelos modelos baseados em fluxo de caixa. Penman (2005) afirma que os diversos modelos de avaliação de empresas devem convergir para um mesmo valor.

No mesmo sentido em que Martins (2001, p. 12), discutindo os métodos de avaliação patrimonial, considera que "no longo prazo, todos são nada mais nada menos do que distribuições temporais diferentes do mesmíssimo fluxo físico de caixa. Assim, no longo prazo, são todos convergentes para um único valor de lucro [...]". Nesse sentido, os modelos fundamentados em fluxos de caixa e os baseados em fluxos de lucro deveriam estimar valores convergentes para as empresas.

O objetivo do presente estudo é examinar se os valores das empresas estimados pelos modelos com maior embasamento na contabilidade, o RIV e o AEG, se aproximam dos valores estimados pelo Fluxo de Caixa Descontado reportados nos laudos de avaliação das Ofertas Públicas de Aquisição de Ações.

Neste estudo, foram examinados 97 laudos de OPAs disponibilizados no website da Comissão de Valores Mobiliários (CVM) no período de 2000 a 2007. Alguns laudos continham a avaliação de mais de uma empresa, perfazendo uma amostra de 124 firmas avaliadas pelo 
modelo de Fluxo de Caixa Descontado. O modelo RIV foi aplicado em 21 empresas e o AEG em 39 firmas da amostra.

Os resultados obtidos indicam que não existe diferença estatisticamente significante entre os valores das empresas estimados pelos modelos com maior embasamento na contabilidade e os valores estimados pelo Fluxo de Caixa Descontado disponíveis nos laudos de avaliação. Complementarmente, ressalte-se que alguns laudos não cumpriram todas as exigências da CVM, bem como muitos procedimentos de avaliação não são claramente explicados aos usuários que se utilizam de tais relatórios.

Os resultados deste estudo ampliam a literatura sobre avaliação de empresas e são úteis para que os profissionais que elaboram os laudos de avaliação possam agregar novos modelos em seus trabalhos, bem como, para que pesquisadores desenvolvam novas metodologias ou aplicações de tais modelos.

O artigo está estruturado da seguinte forma: na seção 2, é apresentado o referencial teórico que embasa o estudo e as hipóteses de pesquisa; a seção 3 descreve os procedimentos metodológicos utilizados no desenvolvimento do trabalho; já a seção 4 apresenta os resultados obtidos e, por fim, na seção 5, têm-se as considerações finais do estudo.

\section{PLATAFORMA TEÓRICA E HIPÓTESES DE PESQUISA}

As companhias abertas que realizam OPAs para fechamento de capital, alienação do controle acionário e participação em fusões e aquisições, entre outros propósitos, contratam profissionais para elaborar um laudo de avaliação em conformidade com as normas em vigor.

Um dos objetivos dos laudos de avaliação é a redução da assimetria de informações entre os agentes internos e externos interessados no valor da firma. Os laudos de avaliação são elaborados por profissionais especializados com atributos definidos em dispositivo normativo. Em se tratando de Ofertas Públicas de Aquisição de Ações, a referência normativa principal é a Instrução CVM 361/2002, que rege as exigências a serem cumpridas na realização da proposta ao mercado e na elaboração do laudo das OPAs.

O artigo $8^{\circ}$ da Instrução 361/2002 da CVM estabelece as abordagens que devem ser utilizadas no processo de avaliação de empresas, de forma que os resultados obtidos pelos diversos modelos existentes possam servir de base para a tomada de decisão dos acionistas que receberem proposta para a venda de suas ações:

I - preço médio ponderado de cotação das ações da companhia objeto na bolsa de valores ou no mercado de balcão organizado, nos últimos 12 meses, se houver, discriminando os preços das ações por espécie e classe;

II - valor do patrimônio líquido por ação da companhia objeto apurado nas últimas informações periódicas enviadas à CVM;

III - valor econômico da companhia objeto por ação, calculado pela regra do fluxo de caixa descontado ou por múltiplos, conforme se entender fundamentadamente mais adequado ao caso da companhia, de modo a avaliá-la corretamente;

IV - valor da companhia segundo o critério de avaliação adotado pelo ofertante para a definição do preço justo, se for o caso, e não estiver abrangido nos incisos anteriores. 
Entre as diversas abordagens indicadas pela CVM, fica em aberto, no inciso IV, a aplicação de outros modelos para verificar a consistência dos valores obtidos. Martins (2001) ressalta que dos diversos modelos que podem ser utilizados para avaliar uma empresa, o teoricamente ideal é o que está atrelado ao propósito da avaliação e às características dos negócios.

A obrigação pela utilização de determinados modelos de avaliação de empresas pode não ser compatível com a real situação econômica da firma avaliada (SANTOS et al., 2008), o que não consideraria as especificidades do negócio e a limitação para a utilização de modelos alternativos aos tradicionalmente utilizados pelos agentes do mercado.

Pesquisas recentes que examinaram os laudos de OPAs no Brasil tiveram como foco a verificação do cumprimento dos dispositivos legais dos relatórios e não a análise dos modelos de avaliação e a proposição de métodos alternativos (SANTOS et al., 2008; IZECKSOHN NETO, 2008).

Santos et al. (2008) procuraram mensurar o grau de cumprimento das exigências da CVM em relação ao que deve constar dos laudos de avaliação no período de 2003 a 2007. Suas conclusões indicaram que os laudos em geral não seguem as exigências legais dos órgãos reguladores. No entanto, fica em aberto a discussão sobre aplicação dos dados utilizados em outros modelos para verificar a consistência dos valores obtidos, principalmente pelo modelo de Fluxo de Caixa Descontado.

Izecksohn Neto (2008) destaca, entre outros aspectos, que todos os modelos utilizados nos laudos analisados em sua pesquisa levam a problemas práticos para reembolsar os acionistas minoritários, pois o valor econômico da empresa possui elevada subjetividade podendo levar a potenciais conflitos de interesse. Além disso, o valor patrimonial e o preço médio ponderado apresentaram alta volatilidade.

Outro fator importante é que o processo de avaliação não depende somente da teoria e dos modelos, mas também da experiência de cada avaliador, suas premissas, raciocínio lógico, capacidade cognitiva e valores pessoais que podem construir os atributos necessários para cruzar um emaranhado de informações contábeis, financeiras e estatísticas, bem como micro e macro econômicas e, igualmente, flutuações estruturais dos fundamentos das economias (NEIVA, 1992; IZECKSOHN NETO, 2008).

Izecksohn Neto (2008, p.1) argumenta que "a principal ferramenta do analista na tarefa de tentar adivinhar o futuro para obter lucros advém das publicações financeiras de cada companhia, sob a forma de seus balanços patrimoniais, demonstrações de resultados, notas explicativas [...]". Dessa forma, constata-se a importância da informação contábil no desenvolvimento dos laudos e como elemento fundamental para a redução da assimetria informacional.

Santos et al. (2008, p.13) destacam que, quando o agente regulador especifica os modelos de avaliação, "a teoria científica não deve ser vinculada a normas e seus fundamentos nem sempre têm relação com desejos ou intenções de órgãos reguladores por melhor intenção que julguem ter". Dessa maneira, pode ser uma evidência da não aplicação de modelos alternativos aos destacados pelos dispositivos legais no mercado acionário brasileiro.

A comparação entre modelos RIV, AEG e Fluxo de Caixa Descontado foi amplamente realizada na literatura internacional (PENMAN, 2005). No entanto, conforme discutido, no Brasil existem poucos estudos sobre a proximidade ou não dos valores estimados por esses modelos, o que poderia ser diferente de outros países considerando a eficiência e regulação de cada mercado de capitais.

Um dos primeiros estudiosos que analisou a aplicabilidade do RIV, no Brasil, foi 
Lopes (2001), no entanto, Sant'anna (2004) comparou os modelos RIV e AEG. Os resultados do trabalho não indicaram superioridade entre os modelos com base no poder explicativo das regressões, exceto para dois períodos específicos. O autor ressalta que tais evidências podem estar relacionadas com a estrutura de governança corporativa das firmas, pelas características do mercado bank-oriented system e por aspectos tributários.

Galdi e Lopes (2006) compararam os modelos Fluxo de Caixa Descontado e RIV, utilizando as projeções de analistas e não dados históricos como, até então, era normalmente feito. Os autores evidenciaram diferenças estatisticamente significantes entre os valores estimados pelos modelos, bem como o Índice Preço/PL calculado pelas informações dos analistas sobre o fluxo de caixa tem maior poder explicativo do que quando estimado pelo modelo RIV.

Ohlson e Lopes (2007) fizeram uma integração dos diversos modelos de avaliação de empresas, partindo do modelo de dividendos de Gordon, passando pelo Fluxo de Caixa Descontado, RIV e AEG. Os autores argumentam que o modelo AEG é, teoricamente, superior aos demais e discutem a validade dos modelos baseados em números contábeis para o mercado. Ainda enfatizam que evidências, em mercados como o Brasil, contribuiriam para a validade e a utilidade de tais modelos.

Ferreira (2008) analisou a relevância da informação entre os modelos RIV, AEG e Fluxo de Caixa Livre no período entre 1995 e 2002. Os resultados indicaram maior relevância do modelo RIV no período entre 1995 e 1999. Já, entre 2000 e 2002, os modelos RIV e AEG se equivalem quanto ao poder explicativo. O Fluxo de Caixa Livre apresentou, em todas as janelas de tempo, menor poder explicativo medido pelo $\mathrm{R}^{2}$, porém, o estudo não examinou a diferença entre os valores estimados em cada modelo, mas, sim, o poder explicativo das regressões em relação ao preço da ação.

$\mathrm{Na}$ estrutura legal e financeira no Brasil, a contabilidade é utilizada para arranjos contratuais por meio de covenants para assegurar o retorno dos financiadores de capital. Além disso, há uma forte relação entre a contabilidade e o sistema tributário, devido à intervenção da Receita Federal do Brasil nas normais contábeis. Em consequência, os modelos de avaliação baseados em lucros contábeis poderiam ser impactados por tais fatores e se distanciarem do valor econômico da empresa.

Cabe destacar, entretanto, que existe uma ligação entre o lucro contábil e o fluxo de caixa das empresas. O lucro elaborado à luz do regime de competência de exercícios nada mais é do que a simples distribuição temporal lógica dos fluxos de caixas. Em algum momento do tempo, o lucro transitará pelo caixa da empresa.

Nesse sentido, Martins (1999, p.13-14) comenta que "no caso extremo, qual o lucro de uma empresa no longo prazo? É a diferença entre o valor de sua liquidação e os investimentos feitos pelos sócios (considerando-se, é claro, os lucros distribuídos durante a sua vida). Assim, o conceito tradicional e intuitivo de lucro está vinculado, em sua última conseqüência, ao fluxo financeiro de ponta a ponta entre a empresa e o seu proprietário."

Ainda que, no curto prazo, ocorram diferenças materiais entre o lucro e o fluxo de caixa, no longo prazo, essas variáveis devem convergir para um mesmo valor. O processo de avaliação, em geral, considera a empresa em situação de continuidade, projetando lucros ou fluxos de caixa no longo prazo (perpetuidade). Em conseqüência, espera-se que tanto os modelos de avaliação baseados em fluxos de caixa quanto os baseados em lucros devem estimar valores próximos para as empresas, uma vez que as variáveis subjacentes a esses modelos, lucro e fluxo de caixa, são convergentes no longo prazo. 
Nesse contexto, a presente pesquisa procura verificar a proximidade dos valores estimados por meio de modelos de Fluxo de Caixa Descontado, como o fluxo de caixa livre da empresa e o fluxo de caixa livre do acionista, e por modelos com maior embasamento na contabilidade, o RIV e o AEG. O estudo examina as seguintes hipóteses de pesquisa:

$H_{1}$ : os valores das empresas estimados pelo modelo Residual Income Valuation se aproximam dos valores estimados nos laudos de avaliação pelo método do Fluxo de Caixa Descontado.

$\mathrm{H}_{2}$ : os valores das empresas estimados pelo modelo Abnormal Earnings Growth se aproximam dos valores estimados nos laudos de avaliação pelo método do Fluxo de Caixa Descontado.

Os modelos de avaliação com base em números contábeis considerados nesta pesquisa foram estruturados, principalmente, por Ohlson (1995) e Ohlson (2005). O autor construiu um arcabouço teórico e analítico que estima o valor da firma em função de variáveis contábeis como o lucro e o patrimônio líquido.

No modelo RIV, o valor da empresa corresponde ao seu patrimônio líquido atual somado ao valor presente dos fluxos futuros de lucros residuais (ou anormais). O lucro anormal é aquele que excede ao custo de oportunidade do capital próprio investido (PL) na empresa.

O modelo RIV é dado por:

$$
P_{0}=P L_{0}+\sum_{t=1}^{\infty} \frac{E\left(\text { Lucro }_{t}^{a}\right)}{(1+r)^{\dagger}} \quad \text { (equação 1) }
$$

em que:

- $\mathrm{P}_{0}$ : valor da empresa na data zero;

- $\mathrm{PL}_{0}$ : patrimônio líquido na data zero;

- $\quad$ r: custo de oportunidade do capital;

- Lucro $_{\mathrm{t}}^{\mathrm{a}}$ : lucro anormal no período t $\left(\right.$ lucro $\left._{\mathrm{t}}-\mathrm{r} \cdot \mathrm{PL}_{\mathrm{t}-1}\right)$.

Detalhes da estrutura e da utilização do modelo RIV podem ser obtidos no artigo de Cupertino e Lustosa (2004) que desenvolveram um tutorial para aplicação.

Avançando na pesquisa sobre valuation, Ohlson (2005) propôs um aprimoramento do modelo RIV para um novo modelo baseado somente no lucro líquido, motivado pelo fato de que os analistas, muitas vezes, projetam somente o lucro da empresa e não o patrimônio líquido.

Nesse modelo, denominado AEG, o valor da empresa é dado pela perpetuidade do lucro líquido projetado para o primeiro ano, somado ao valor presente da expectativa de crescimento anormal do lucro líquido. 
Já o modelo AEG é dado por:

$$
P_{0}=\frac{\text { lucro }_{1}}{r}+\sum_{t=1}^{\infty} \frac{\left[\frac{1}{r}\left(\text { (ulucro }_{t+1}-r \cdot\left(\text { lucro }_{t}-\text { div }_{t}\right)\right)\right]}{(1+r)} \quad \text { (equação 2) }
$$

em que:

- $\mathrm{P}_{0}$ : valor da empresa na data zero;

- $r$ : custo de oportunidade do capital;

- lucro: lucro na data $t$;

- $\operatorname{div}_{\mathrm{t}}$ : dividendo na data $\mathrm{t}$.

Calijuri et al. (2008) desenvolveram um tutorial para a aplicação do modelo AEG, bem como detalhes da estrutura e da utilização.

Ohlson (2005) e Penman (2005) mostraram a equivalência e a convergência de ambos os modelos analiticamente. No entanto, Penman (2005) destaca que o AEG, inicialmente, sofre efeitos nos valores estimados devido ao fato de que os analistas são mais otimistas no curto prazo, embora, no longo prazo, os valores se ajustarão ou pelo menos deverão se equiparar.

A equivalência dos modelos dá-se pelo fato de ambos possuírem um valor âncora de partida. No caso do RIV, é o patrimônio líquido inicial $\left(\mathrm{PL}_{0}\right)$ e, para o $\mathrm{AEG}$, é a perpetuidade (taxa que se equivalha ao custo de capital do PL) do lucro projetado para o primeiro período. Dessa forma, $\mathrm{PL}_{0}=$ lucro $_{1} / \mathrm{r}$. No modelo RIV, precisa ser respeitada a premissa da clean surplus relation, que impõe que todas as transações que modifiquem o patrimônio líquido da empresa, exceto as realizadas com os acionistas, devem transitar pelas contas de resultado. Já no modelo AEG, essa premissa não é assumida (OHLSON e LOPES, 2007).

Ohlson e Lopes (2007) afirmam que "acreditamos que o RIV nunca atingirá o status de estrutura de avaliação principal pelo fato de que ela focaliza, essencialmente, o patrimônio líquido corrente e seu subseqüente crescimento. O importante realmente é o lucro e seu crescimento [...]". Nesse sentido, a aplicação do RIV mostra-se mais complexa em relação a outras modelagens.

\section{MÉTODO DE PESQUISA}

A presente pesquisa examina se os valores das empresas estimados por meio dos modelos RIV e AEG se aproximam dos valores estimados pelo modelo de Fluxo de Caixa Descontado. O estudo foi realizado com base nos dados dos laudos de avaliação constantes das OPAs, obtidos no site da CVM. O procedimento inicial foi analisar cada laudo individualmente para a coleta dos dados dos fluxos de caixa, de lucros, patrimônio líquido, taxas de desconto e taxas livre de risco, para, posteriormente, aplicar os modelos RIV e AEG e os testes de média.

A amostra selecionada compreende 97 laudos de avaliação que foram publicados no período entre 2000 e 2007. Como alguns laudos envolvem a avaliação de mais de uma empresa, a amostra final utilizada no estudo perfez um total de 148 firmas. A tabela 1 exibe a quantidade de laudos de avaliação utilizados por ano do período de pesquisa. 
Tabela 1: Quantidade de Laudos de Avaliação

\begin{tabular}{c|c}
\hline Ano & Quantidade de Laudos \\
\hline 2000 & 1 \\
\hline 2001 & 1 \\
\hline 2002 & 18 \\
\hline 2003 & 16 \\
\hline 2004 & 25 \\
\hline 2005 & 9 \\
\hline 2006 & 18 \\
\hline 2007 & 9 \\
\hline Total & 97 \\
\hline
\end{tabular}

A maior parte dos laudos de avaliação incluídos na amostra $(56,7 \%)$ teve por objetivo a realização de OPA para cancelamento de registro de companhia aberta (fechamento de capital). Outros objetivos identificados nos laudos de avaliação são: alienação de controle, leilão para desestatização, permuta de ações, recompra de ações e reestruturações societárias.

A análise dos laudos que compõem a amostra evidenciou que os avaliadores utilizam diversos métodos para estimar o valor das empresas. Em alguns casos, a avaliação é feita com base em apenas um modelo, no entanto, na maioria dos casos, mais de um método foi aplicado para se obter o valor de cada firma.

A tabela 2 exibe a quantidade de empresas às quais foram aplicados os principais métodos de avaliação constantes dos laudos:

Tabela 2: Métodos de Avaliação Utilizados

\begin{tabular}{l|c}
\hline \multicolumn{1}{c|}{ Método de Avaliação } & Quantidade de Empresas \\
\hline Fluxo de Caixa Descontado & 124 \\
\hline Valor Contábil das Ações & 113 \\
\hline Valor de Mercado das Ações & 96 \\
\hline Múltiplos de Mercado & 55 \\
\hline Valor de Mercado dos Itens Patrimoniais & 8 \\
\hline
\end{tabular}

Como se pode observar, o método de avaliação mais utilizado é o Fluxo de Caixa Descontado, que foi aplicado em 124 das 148 empresas avaliadas (83,8\%). O segundo método de avaliação mais utilizado foi o valor contábil das ações (76,4\% das empresas). Verifica-se, também, que outros modelos como o RIV e AEG não foram utilizados em nenhum dos laudos analisados.

Limitou-se a amostra aos laudos que apresentavam a aplicação do Fluxo de Caixa Descontado bem como a projeção do lucro líquido e patrimônio líquido, variáveis necessárias para o RIV e AEG, já que o objetivo está na comparação entre os valores estimados pelos três modelos. Essa restrição levou a uma redução na quantidade de empresas da amostra, uma vez que muitos laudos de avaliação não contêm dados de lucro líquido e patrimônio líquido. Então, não necessariamente, a mesma empresa consta das duas amostras. A tabela 3 exibe a quantidade 
de empresas cujos laudos forneceram informações suficientes para a aplicação dos modelos RIV e AEG.

Tabela 3: Quantidade de Empresas Avaliadas

\begin{tabular}{c|c}
\hline \multicolumn{1}{c|}{ Método de Avaliação } & Quantidade de Empresas \\
\hline Residual Income Valuation & 21 \\
\hline Abnormal Earnings Growth & 39 \\
\hline
\end{tabular}

Cabe destacar que a aplicação do modelo RIV foi feita por meio de dois procedimentos alternativos. O primeiro procedimento considerou a variável lucro como a variação do patrimônio líquido projetado (Lucro $=\mathrm{PL}_{\mathrm{t}}-\mathrm{PL}_{\mathrm{t}-1}$ ), enquanto o segundo considerou a variável lucro como o próprio lucro líquido projetado.

Essas duas formas de estimar o resultado contábil utilizado no RIV são feitas para checar a funcionalidade da CSR, conforme os dados projetados nos laudos, ou seja, tecnicamente, se todas as movimentações das firmas forem transacionadas pelo resultado, o valor da variação patrimonial deverá ser igual ao lucro líquido. Então, optou-se por utilizar as duas variáveis para dar mais robustez aos resultados.

Para testar as hipóteses de pesquisa, foi examinado se há diferenças estatisticamente significantes entre os valores estimados pelo modelo Fluxo de Caixa Descontado e os valores estimados pelo RIV e AEG.

Os valores do modelo Fluxo de Caixa Descontado são calculados pelos próprios avaliadores e reportados nos laudos de avaliação. Já os valores dos modelos RIV e AEG foram estimados por meio da aplicação das equações 1 e 2 aos dados de patrimônio líquido e lucro líquido disponibilizados nos laudos. Se os valores estimados pelos modelos não apresentarem diferenças estatisticamente significantes, serão considerados como próximos. Nesse caso, os modelos de avaliação de empresas baseados em números contábeis produziriam valores próximos aos gerados pelo Fluxo de Caixa Descontado.

O exame da significância estatística das diferenças entre os valores gerados pelos modelos é feito por meio da aplicação de testes de médias. Como os testes paramétricos se baseiam em suposições acerca das distribuições de probabilidade das variáveis em estudo, é necessário examinar se essas variáveis são normalmente distribuídas previamente à aplicação dos testes.

O teste de aderência de Kolmogorov-Smirnov (KS) foi empregado para examinar se as variáveis têm distribuição normal. O teste analisa o grau de aderência entre a distribuição de um conjunto de valores observados e determinada distribuição teórica específica, nesse caso, a distribuição normal. Ademais, determina se os valores da amostra podem, razoavelmente, ser considerados como provenientes de uma população com a distribuição teórica especificada (BUSSAB e MORETTIN, 2002)

A hipótese nula do teste KS considera que a amostra foi extraída da distribuição teórica especificada, ou seja, espera-se que a diferença entre a distribuição de freqüência acumulada da amostra, $\mathrm{F}_{\mathrm{o}}(\mathrm{X})$ e a distribuição de freqüência acumulada teórica sob $\mathrm{H}_{0}, \mathrm{~F}_{\mathrm{e}}(\mathrm{X})$ sejam pequenas e estejam dentro dos limites dos erros aleatórios. O teste focaliza a maior dessas diferenças absolutas, $\left|\mathrm{F}_{\mathrm{e}}(\mathrm{X})-\mathrm{F}_{\mathrm{o}}(\mathrm{X})\right|$, que é chamada de desvio máximo (D).

Se as variáveis em estudo não forem normalmente distribuídas, a utilização de testes 
de média paramétricos não é adequada. Nesse caso, as diferenças são examinadas por meio de testes não-paramétricos, que não se baseiam em suposições sobre os parâmetros da população.

Um teste não-paramétrico utilizado para examinar diferenças entre médias de amostras relacionadas é o de Wilcoxon. No presente estudo, as amostras são consideradas relacionadas, uma vez que se trata de valores atribuídos às mesmas empresas por meio da aplicação de modelos de avaliação diferentes, portanto, não podem ser consideradas como amostras independentes.

O teste de Wilcoxon examina o sentido e o valor das diferenças dentro de cada par de observações. O teste atribui maior ponderação ao par que acusar grande diferença entre as condições e menor ponderação àquele em que essa diferença for pequena (CONOVER, 1971). A estatística do teste de Wilcoxon (Z) é dada por:

em que:

$$
Z=\frac{T-\frac{N(N+1)}{4}}{\frac{\sqrt{N(N+1)(2 N+1)}}{24}} \quad \text { (equação 3) }
$$

T: é a menor da soma de postos de mesmo sinal;

$\mathrm{N}$ : é a quantidade de objetos em postos.

\section{ANÁLISE DOS RESULTADOS}

Previamente à apresentação e análise dos resultados dos testes de diferença de média, apresentam-se as estatísticas descritivas dos valores estimados em cada modelo de avaliação em estudo. A tabela 4 exibe as estatísticas descritivas dos valores obtidos com a aplicação dos modelos Fluxo de Caixa Descontado (FCD) e RIV, tanto pelo método da variação do patrimônio líquido projetado (RIV-PL) quanto pelo método do lucro líquido projetado (RIV-LL) devido à CSR como já explicitado.

Tabela 4: Estatísticas Descritivas - FCD e RIV (R\$)

\begin{tabular}{c|c|c|c}
\hline & FCD & RIV-PL & RIV-LL \\
\hline Mínimo & -33 & -1 & 1 \\
\hline Máximo & 1.205 & 1.478 & 1.482 \\
\hline Média & 155 & 132 & 124 \\
\hline Desvio-Padrão & 358 & 347 & 333 \\
\hline
\end{tabular}

As estatísticas descritivas mostram que os valores estimados pelo RIV, tanto pelo método da variação do patrimônio líquido quanto pelo método do lucro líquido, em média, são inferiores aos valores estimados pelo modelo Fluxo de Caixa Descontado.

A tabela 5 exibe as estatísticas descritivas dos valores obtidos com a aplicação dos modelos Fluxo de Caixa Descontado (FCD) e AEG. 
Tabela 5: Estatísticas Descritivas - FCD e AEG (R\$)

\begin{tabular}{c|c|c}
\hline & FCD & AEG \\
\hline Mínimo & -44 & -983 \\
\hline Máximo & 5.724 & 5.776 \\
\hline Média & 1.316 & 1.217 \\
\hline Desvio-Padrão & 1.669 & 1.691 \\
\hline
\end{tabular}

As estatísticas descritivas mostram que os valores estimados pelo modelo AEG, em média, são inferiores aos valores estimados pelo modelo do Fluxo de Caixa Descontado, mas com um desvio-padrão maior mostrando maior volatilidade.

Previamente à realização dos testes de diferença de médias, o teste de aderência de Kolmogorov-Smirnov foi aplicado para examinar se as variáveis em estudo têm distribuição normal de probabilidades. O teste foi aplicado aos valores estimados por cada modelo e também às diferenças entre eles.

A tabela 6 exibe os resultados do referido teste para as empresas avaliadas pelos modelos Fluxo de Caixa Descontado (FCD) e RIV.

Tabela 6: Teste de Kolmogorov-Smirnov - FCD e RIV

\begin{tabular}{l|c|c|c|c|c}
\hline & FCD & RIV-PL & RIV-LL & $\begin{array}{c}\text { Diferença } \\
\text { FCD e RIV-PL }\end{array}$ & $\begin{array}{c}\text { Diferença } \\
\text { FCD e RIV-LL }\end{array}$ \\
\hline Kolmogorov-Smirnov & 1,915 & 1,863 & 1,736 & 2,020 & 1,979 \\
\hline Valor p & 0,001 & 0,002 & 0,005 & 0,001 & 0,001 \\
\hline
\end{tabular}

Os resultados do teste evidenciam que, em todos os casos, ao nível de significância de $5 \%$, rejeita-se a hipótese nula de que as variáveis seguem uma distribuição normal de probabilidades, indicando a aplicação de teste não-paramétrico.

A tabela 7 exibe os resultados do teste de Kolmogorov-Smirnov para as empresas avaliadas pelos modelos Fluxo de Caixa Descontado (FCD) e AEG.

Tabela 7: Teste de Kolmogorov-Smirnov - FCD e AEG

\begin{tabular}{l|c|c|c}
\hline & FCD & AEG & $\begin{array}{c}\text { Diferença } \\
\text { FCD e AEG }\end{array}$ \\
\hline Kolmogorov-Smirnov & 1,378 & 1,556 & 1,865 \\
\hline Valor p & 0,045 & 0,016 & 0,002 \\
\hline
\end{tabular}

Assim como anteriormente, os resultados do teste, também, indicam que, em todos os casos, ao nível de significância de 5\%, rejeita-se a hipótese nula de que as variáveis seguem uma distribuição normal de probabilidades.

Dessa forma, como as variáveis não são normalmente distribuídas, a significância estatística das diferenças entre os valores estimados pelos modelos de avaliação foi examinada 
por meio do teste de Wilcoxon (não-paramétrico). A tabela 8 exibe os resultados do teste de Wilcoxon aplicados aos valores estimados pelos modelos Fluxo de Caixa Descontado (FCD), RIV e AEG.

Tabela 8: Teste de Wilcoxon - FCD, RIV e AEG

\begin{tabular}{l|c|c|c}
\hline & FCD e RIV-PL & FCD e RIV-LL & FCD e AEG \\
\hline Wilcoxon $(\mathrm{Z})$ & $-1,811$ & $-1,130$ & $-0,502$ \\
\hline Valor $\mathrm{p}$ & 0,070 & 0,259 & 0,615 \\
\hline
\end{tabular}

Conforme se observa, os resultados do teste de Wilcoxon indicam que as diferenças entre os valores estimados pelos modelos Fluxo de Caixa Descontado e RIV, tanto pelo método da variação do patrimônio líquido quanto pelo método do lucro líquido, não são, estatisticamente significantes ao nível de 5\%. Da mesma forma, os resultados indicam que as diferenças entre os valores estimados pelo Fluxo de Caixa Descontado e pelo AEG, também, não são, estatisticamente, significantes ao nível de 5\%.

Portanto, há evidências de que os valores estimados pelos modelos RIV e AEG não são, estatisticamente, diferentes dos valores estimados pelo modelo Fluxo de Caixa Descontado. Em consequência, considera-se que os valores estimados por esses modelos são próximos, o que leva à confirmação das hipóteses de pesquisa conforme a abordagem teórica dos modelos.

Para completar a análise, apresenta-se a distribuição das diferenças entre os valores estimados pelos modelos. A tabela 9 exibe as diferenças entre os valores estimados pelos modelos Fluxo de Caixa Descontado (FCD) e RIV em três faixas.

Tabela 9: Distribuição das Diferenças: RIV-PL - FCD e RIV-LL - FCD

\begin{tabular}{c|c|c|c|c}
\hline \multirow{2}{*}{ Diferença } & \multicolumn{2}{|c|}{ RIV-PL } & \multicolumn{2}{c}{ RIV-LL } \\
\cline { 2 - 5 } & $\begin{array}{c}\text { Quantidade de } \\
\text { Empresas }\end{array}$ & $\%$ & $\begin{array}{c}\text { Quantidade de } \\
\text { Empresas }\end{array}$ & $\%$ \\
\hline Inferior a -20\% & 10 & 47,6 & 12 & 57,1 \\
\hline Entre -20\% e 20\% & 8 & 38,1 & 8 & 38,1 \\
\hline Superior a 20\% & 3 & 14,3 & 1 & 4,8 \\
\hline Total & $\mathbf{2 1}$ & $\mathbf{1 0 0 , 0}$ & $\mathbf{2 1}$ & $\mathbf{1 0 0 , 0}$ \\
\hline
\end{tabular}

Das 21 empresas para as quais foi possível a aplicação do modelo RIV, em 8 empresas $(38,1 \%$ da amostra) a diferença entre os valores produzidos por esse modelo e pelo Fluxo de Caixa Descontado está entre $-20 \%$ e $20 \%$; nas outras extremidades, o número de firmas se alteram sugerindo que, em tais avaliações, a CSR não foi respeitada plenamente nas projeções.

Em $47,6 \%$ e $57,1 \%$ das empresas, respectivamente, os modelos RIV-PL e RIV-LL estimam valores com diferenças inferiores a $-20 \%$, mostrando que esse modelo, para a amostra analisada, apresenta uma tendência de subestimação do valor da empresa em relação ao Fluxo de Caixa Descontado.

Essa evidência pode estar relacionada com dois fatores levantados pela literatura internacional: i) o argumento de Penman e Sougiannis (1998) de que o conservadorismo 
na contabilidade pode afetar os cálculos de modelos e pode ser observado nos múltiplos de patrimônio líquido e lucro divididos pelo preço, sendo assim, modelos baseados em números contábeis podem ter performance menor quando o modelo do padrão contábil está associado a uma contabilidade conservadora; e ii) a não correção do conservadorismo na série de projeções dos lucros residuais, que conforme Choi, O'Hanlon e Pope (2006) propuseram uma correção no modelo RIV que procura corrigir o conservadorismo das projeções dos lucros residuais, adicionando outras informações nesses fluxos projetados que mitiga os efeitos do conservadorismo contábil. Os resultados do estudo indicam que a correção dos efeitos do conservadorismo aumenta a performance do modelo no que tange o viés, mas não reduz a baixa acurácia dos valores estimados.

A tabela 10 exibe as diferenças entre os valores estimados pelos modelos Fluxo de Caixa Descontado (FCD) e AEG em três faixas.

Tabela 10: Distribuição das Diferenças: AEG - FCD

\begin{tabular}{c|c|c}
\hline \multicolumn{1}{c|}{ Diferença } & Quantidade de Empresas & \% \\
\hline Inferior a -20\% & 15 & 38,5 \\
\hline Entre -20\% e 20\% & 10 & 25,6 \\
\hline Superior a 20\% & 14 & 35,9 \\
\hline Total & $\mathbf{3 9}$ & $\mathbf{1 0 0 , 0}$ \\
\hline
\end{tabular}

Das 39 empresas em que foi possível a aplicação do modelo AEG, em 10 empresas (25,6\% da amostra) a diferença entre os valores produzidos por esse modelo e pelo Fluxo de Caixa Descontado está entre $-20 \%$ e $20 \%$.

Em 38,5\% das empresas, o modelo AEG estima valores com diferenças inferiores a $-20 \%$ em relação ao Fluxo de Caixa Descontado, enquanto, em 35,9\% das empresas, o modelo AEG estima valores com diferenças superiores a $20 \%$. Nesse caso, não se verifica tendência de subestimação ou de superestimação do valor da empresa por parte do modelo AEG em relação ao Fluxo de Caixa Descontado.

Essas dispersões, apresentadas nas tabelas 9 e 10, contribuem para ilustrar que a aplicação de cada modelo depende da situação específica de cada firma e que a mera atenção ao cumprimento dos dispositivos legais também podem interferir para encontrar o valor econômico para os acionistas.

\section{CONSIDERAÇÕES FINAIS}

Os resultados dos testes empíricos realizados evidenciam que, para as empresas que fizeram parte da amostra, os valores estimados por meio dos modelos RIV e AEG são próximos aos valores estimados pelo Fluxo de Caixa Descontado disponíveis nos laudos de avaliação das OPAs.

Em outras palavras, a partir dos dados coletados nos laudos de avaliação e dos testes 
efetuados, não foram encontradas diferenças estatisticamente significantes entre os valores estimados pelos modelos com base em números contábeis e pelo modelo baseado no fluxo de caixa. Essas evidências levam à confirmação das hipóteses de pesquisa.

Esse resultado é decorrente do conceito de que no longo prazo o lucro e o fluxo de caixa convergem para o mesmo valor, conforme amplamente discutido por Martins (1999), Martins (2001). No curto prazo, porém, diferenças devem ser observadas entre os modelos Fluxo de Caixa Descontado, RIV e AEG, devido às características do sistema contábil baseado no regime de competência.

Cabe ressaltar, também, que a comparação direta entre os modelos RIV e AEG não foi possível neste trabalho, uma vez que os referidos modelos foram aplicados em empresas diferentes em cada amostra.

A contribuição prática da pesquisa é que os modelos RIV e AEG poderiam ser utilizados de forma alternativa nos processos de elaboração de laudos de Oferta Pública de Aquisição de Ações, em conformidade com o inciso IV, parágrafo terceiro, artigo $8^{\circ}$, da Instrução CVM n ${ }^{\circ}$ 361/2002 que, ao invés de fazer restrições, deixa em aberto a possibilidade de aplicação de modelos alternativos ou complementares.

Adicionalmente, ressalte-se que muitos laudos não cumpriram todas as exigências normativas da Comissão de Valores Mobiliários, bem como muitos procedimentos não são claramente explicados para os usuários dos relatórios, além da falta de uma padronização dos laudos de avaliação, no entanto, ao longo do tempo foram ficando mais completos.

Para pesquisas futuras são sugeridas análises qualitativas das principais variações ocorridas, talvez separando as empresas em grupos (clusters). Seria importante uma demonstração das divergências observadas nos valores, tanto para o RIV como para o AEG. A justificativa para esse tipo de estudo é a aplicação desses modelos na prática de avaliações de empresas no país, uma vez que contribuições poderiam ser feitas no sentido de indicar em que situações peculiares os modelos seriam mais adequados.

\section{REFERÊNCIAS}

BUSSAB, W.O; MORETTIN, P.A. Estatística Básica. 5 ed. São Paulo: Saraiva, 2002.

CALIJURI, M.S.S; BISPO, J.S; JUNQUEIRA, E.R. Avaliação de Empresas pelo Modelo Ohlson e Juettner-Nauroth (2005): Tutorial Para Uso. In: $8^{\circ}$ Congresso USP de Contabilidade e Controladoria, 2008.

CHOI, Young-Soo; O'HANLON, John F.; POPE, Peter F., Conservative Accounting and Linear Information Valuation Models. Contemporary Accounting Research, Vol. 23, No. 1, Spring 2006. 
CONOVER, W.J. Practical Nonparametric Statistics. Nova York: Wiley, 1973.

EDWARDS, E; BELL, P.W. The Theory and Measurement of Business Income. University of California Press, California, 1961.

FAMÁ, R; LEITE, E.C. O Modelo de Avaliação de Empresas de Edwards-Bell-Ohlson (EBO) - Aspectos Práticos e Teóricos. In: VI SEMEAD, 2003.

FERREIRA, E.S. Comparação entre os Modelos Residual Income Valuation (RIV), Abnormal Earnings Growth (AEG) e Fluxo de Caixa Livre (FCF): um Estudo Empírico no Mercado de Capitais Brasileiro. 2008. 97 f. Dissertação (Mestrado em Ciências Contábeis) - Programa de Pós-Graduação em Ciências Contábeis, Fundação Instituto Capixaba de Pesquisas em Contabilidade, Economia e Finanças (FUCAPE), Vitória, 2008.

GALDI, F.C; LOPES, A.B. Análise Empírica de Modelos de Valuation no Ambiente Brasileiro: Fluxo de Caixa Descontado versus Modelo de Ohlson. In: XXX Encontro Nacional da Associação Nacional dos Programas de Pós-graduação em Administração EnANPAD. 2006.

IZECKSOHN NETO, D. Valor Econômico, Preço Médio Ponderado ou Valor Patrimonial: Métodos de Avaliação do 'Preço Justo' e o Reembolso aos Minoritários. In: Encontro Nacional da Associação Nacional dos Programas de Pós-graduação em Administração, 32, 2008. São Paulo. Anais... Rio de Janeiro: Rio de Janeiro, 2008. CD-ROM

LOPES, A.B. Uma Contribuição ao Estudo da Relevância da Informação Contábil para o Mercado de Capitais: O Modelo de Ohlson Aplicado à BOVESPA. 2001. Tese (Doutorado em Ciências Contábeis) - Programa de Pós-Graduação em Ciências Contábeis, Departamentos de Contabilidade e Atuária, Faculdade de Economia, Administração e Contabilidade da Universidade de São Paulo, 2001.

LUSTOSA, P.R.B; CUPERTINO, C.M. O Modelo Ohlson de Avaliação de Empresas: Tutorial para Utilização. Brazilian Business Review, v.1, n.1, 2004.

MARTINS, E. Contabilidade vs. Fluxo de Caixa. Caderno de Estudos da Fipecafi, v.11, n.20, 1999.

MARTINS, E. Avaliação de Empresas: da Mensuração Contábil à Econômica. São Paulo: Atlas, 2001.

MARTINS, E; GALDI, F.C; LIMA, G.A.S.F; NECYK, G.A; ABE, C.H.S. Evidências Empíricas de Modelos de Estimação do Custo do Capital Próprio. Brazilian Business Review, v.3, n.2, 2006.

NEIVA, R.A. Valor de Mercado da Empresa: Modelos de Avaliação Econômico-Financeira de Empresas, Exemplos de Avaliação com Cálculos de Valores, Subsídios para Privatização, Compra e Venda, Cisão, Fusão e Incorporação. São Paulo: Atlas, 1992.

OHLSON, J.A. Earnings, Book Values and Dividends in Equity Valuation. Contemporary Accounting Research, v. 11, n. 2, 1995. 
OHLSON, J.A. On Accounting-Based Valuation Formulae. Review of Accounting Studies, v. 10,2005

OHLSON, J.A; JUETTNER-NAUROTH, B.E. Expected EPS and EPS Growth as Determinants of Value. Review of Accounting Studies, v. 10, 2005.

OHLSON, J.A; LOPES, A.B. Avaliação de Empresas com Base em Números Contábeis. Brazilian Business Review, v. 4, n. 2, 2007.

PENMAN, S.H. Discussion. Discussion of "On Accounting-Based Valuation Formulae" and "Expected EPS and EPS Growth as Determinants of Value'. Review of Accounting Studies, v. 10, 2005.

PENMAN, S. H.; SOUGIANNIS, T. A Comparison of Dividend, Cash Flow, and Earnings Approaches to Equity Valuation. Contemporary Accounting Research, v. 15 (3), Fall, p. 343383, 1998.

SANT'ANNA, D.P. A Relevância das Informações Contábeis na BOVESPA: Avaliação dos Modelos de Residual Income Valuation e Abnormal Earnings Growth. 2004. 103 f. Dissertação (Mestrado em Ciências Contábeis) - Programa de Pós-Graduação em Ciências Contábeis, Fundação Instituto Capixaba de Pesquisas em Contabilidade, Economia e Finanças (FUCAPE), Vitória, 2004.

SANTOS, A.C; CAMPOS, E.S; FELIPE, E.S; ANJOS, V.M.L. Ofertas Públicas de Aquisição de Ações de Companhia Aberta (OPA): Investigação dos Laudos de Avaliação, In: Encontro Nacional da Associação Nacional dos Programas de Pós-graduação em Administração, 32, 2008. São Paulo. Anais... Rio de Janeiro: Rio de Janeiro, 2008. CD-ROM. 


\section{DADOS DOS AUTORES:}

\section{JOSÉ ELIAS FERES DE ALMEIDA}

Universidade Federal do Espírito Santo, Centro de Ciências Jurídicas e Econômicas, Departamento de Ciências Contábeis.

Avenida Fernando Ferrari, 514, CCJE, ED-VI, sl. 636. Campus Universitário - Goiabeiras 29075-910 - Vitoria, ES - Brasil

\section{GIOVANI ANTONIO SILVA BRITO}

Fipecafi - Fundação Instituto de Pesquisas Contábeis, Atuariais e Financeiras.

Rua Maestro Cardim, 1170, Paraíso

01323-001 - São Paulo, SP - Brasil

\section{FLÁVIO DONIZETE BATISTELLA}

Universidade de São Paulo, Faculdade de Economia, Administração e Contabilidade de Ribeirão Preto.

Av. dos Bandeirantes, 3.900 Monte Alegre

14040-900 - Ribeirão Preto, SP - Brasil

\section{ELISEU MARTINS}

Universidade de São Paulo, Faculdade de Economia Administração e Contabilidade, Departamento de Contabilidade Atuária.

Av. Professor Luciano Gualberto, 908 Prédio III - Butantã

05508-900 - Sao Paulo, SP - Brasil 